\title{
En torno a la emigración gallega a América en el siglo XIX. Algunas consideraciones a la luz del ejemplo canario
}

\author{
Antonio EIRAS RoEL *
}

Galicia comparte con otros pueblos hispánicos el destino migratorio, al menos desde la época de la emigración en masa que se inicia en el último cuarto del siglo $x \mathrm{X}^{1}{ }^{1}$. Pero es principalmente el archipiélago canario la región que se asimila a Galicia por el carácter masivo de su éxodo, por el arraigo tradicional del mismo, y por las análogas razones de necesidad que lo explican. Desde que se cuenta con fuentes estadísticas que permiten establecer la comparación, las islas Canarias, junto con las provincias occidentales de Galicia, encabezan las listas de las provincias españolas por los índices de su emigración relativa, destacándose netamente de todas las demás ${ }^{2}$. Canarios y gallegos cuentan además con una arraigada tradición emigrante a América, que comienza a adquirir importancia cuantitativa al menos desde el siglo $\mathrm{XVIII}{ }^{3}$. Hay que

* Universidad de Santiago de Compostela.

1 Para una presentación de las regiones españolas más relacionadas con la emigración a América, véase el reciente libro de Nicolás Sánchez Albornoz: Españoles hacia América. La emigración en masa, 1880-1930. Alianza Ed., Madrid 1988.

${ }^{2}$ En el bienio 1885-86 Canarias, Pontevedra y La Coruña encabezan la lista de las provincias españolas según el orden de su emigración relativa (por cada 10.000 habitantes). En el quinquenio 1891-95, Almería y Alicante pasan a ocupar el primer y tercer lugar; pero su emigración, de radio y ciclo corto, va dirigida a Argelia.

${ }^{3}$ Hay que recordar el proyecto del Gobernador de Buenos Aires, Bruno Zabala, en 1725, de poblar con familias gallegas y canarias los territorios de la Banda Oriental, a raiz de la fundación de Montevideo. De este relato se ocupa el conocido trabajo de Luisa Cuesta (1932). 
señalar la ventaja cronológica de la región canaria respecto a la gallega por lo que se refiere al estudio reciente de su emigración, que le permite contar con una obra extensa dedicada enteramente al tema, y otros estudios posteriores ${ }^{4}$. Desde el actual horizonte gallego puede ser provechoso prestar la debida consideración a estos antecedentes canarios conforme a las normas del método comparado y apoyarse en ellos para definir las grandes vertientes temáticas del estudio: las motivaciones de la emigración; las fuentes específicas localmente disponibles y su crítica; las condiciones de los desplazamientos y sus repercusiones sociales; el problema de la emigración clandestina; las fluctuaciones del flujo migratorio y el intento de determinación de las dimensiones del éxodo en cada una de sus fases. Nuestro propósito en estas páginas está orientado casi exclusivamente a plantear algunas consideraciones sobre la emigración gallega en relación con la primera cara del poliedro, las causas de la emigración.

Los trabajos existentes sobre la emigración canaria explican la intensa emigración del archipiélago en los siglos XVIII y XIX por razones concretas de índole económica y social que configuran un cuadro de alta presión demográfica. En último término las razones invocadas se vinculan al elevado crecimiento demográfico del archipiélago; y de un modo más próximo, a las consecuencias económicas y sociales que aquél origina: entre las repercusiones económicas estarían la excesiva división del terrazgo y la carestía de los mantenimientos (interpretada por algunas fuentes de la época como "carencia de los artículos de primera necesidad"); y entre las repercusiones sociales sobresale el sistema de medianeros en el régimen de propiedad canario (que para el campesino supone la existencia de "dos amos", "uno real, el medianero, y otro teórico, el propietario de la finca») ${ }^{5}$. Básicamente, el diagnóstico es común para el caso gallego. Las fuentes oficiales de fines del XIX no dejan de invocar motivaciones demográficas de la emigración gallega, principalmente la «excesiva densidad de población", aunque también aludan como causas permanentes de la costumbre de emigrar al "carácter aventurero de sus habitantes", asi como a la "fecundidad de la raza" (prejuicio éste que las cifras no respaldan) y al deseo de "eludir el servicio militar" ${ }^{6}$. Junto

\footnotetext{
${ }^{4}$ Hernandez Garcia, Julio: La emigración de las islas Canarias en el siglo xix. Las Palmas 1981, 629 páginas. Tesis doctoral realizada bajo la dirección del profesor Antonio Bethencourt.

${ }^{5}$ Hernandez Garcia, J., op. cit., pp. 108 ss.

${ }^{6}$ Estadistica de la emigración e inmigración de España en el quinquenio 1891-1895. Madrid 1898. En este momento la fuente menciona también la existencia de causas coyun-
} 
con los excesivos tributos y el odiado impuesto de consumos, la subdivisión de las tierras, el régimen de propiedad foral y el subforo - variante gallega del sistema canario de medianeros- son contemplados también como causas de la emigración gallega en otros informes de la época reunidos en fuentes oficiales ${ }^{7}$. A este respecto puede observarse una cierta unilateralidad y sesgo en las fuentes referentes a Galicia, que prestan escasa o nula atención a las causas económicas propiamente dichas, como no sea a las de carácter coyuntural y transitorio. Las causas de indole social y política ocupan generalmente el primer plano de la escena en los informes de los sociólogos gallegos que contemplaron de cerca el desencadenamiento del gran éxodo, y que lo achacan al régimen de propiedad de la tierra, a la carga tributaria y a la falta de protección oficial a la agricultura ${ }^{8}$. En cambio las fuentes estadísticas oficiales prescinden más o menos tendenciosamente de este tipo de causas, y se remiten a la presión demográfica para explicar la emigración, tanto en Galicia como en Canarias ${ }^{9}$. Puede apreciarse en estas fuentes estadísticas una matización entre dos formas diferentes de presión demográfica que en las dos regiones conducen a un mismo resultado, el hambre de tierra y la subdivisión extremada de las explotaciones campesinas: en el caso canario la causa estaria en el extraordinario crecimiento demográfico, superior al doble del general español; en el caso gallego se atribuye en cambio a la excesiva densidad de población ${ }^{10}$. De hecho, si se observan los crecimientos diferenciales a través de la tabla de incrementos intercensales de alcance secular, se comprueba que la emigración masiva del siglo XIX, en la que las islas Canarias y Galicia se destacan de modo tan acusado, lo mismo pudo tener efecto con un fuerte crecimiento demográfico, doble del español, en el primer caso, como con un crecimiento demográfico débil e inferior a la media general española, en el segundo caso ${ }^{11}$.

turales, a saber, la pérdida de riqueza pecuaria (Lugo), las malas cosechas (Pontevedra) y la crisis vitícola causada por la filoxera (Orense).

${ }^{7}$ La crisis agricola y pecuaria. Madrid 1889. Intorme de la comisión parlamentaria creada en 1887 para el estudio del problema de la emigración.

\& Brañas, Alfredo: La crisis económica en la época presente y la descentralización regional. Santiago 1892. VALES FAlLDE, J.: La emigración gallega. Madrid 1902.

9 También la Real Orden, de 16 de septiembre de 1853 , que concede la libertad de emigrar tuvo presente el problema canario "para dar conveniente salida al exceso de población de dichas islas". Nada se dice del problema gallego, que era del mismo orden.

${ }^{10}$ Comisión Especial para estudiar los medios de contener en lo posible la emigración por medio del desarrollo del trabajo. Memoria presentada al señor ministro de Fomento. Madrid 1882.

1 La cifra de población para las Canarias en 1650 está tomada de A. Bethencourt: La 


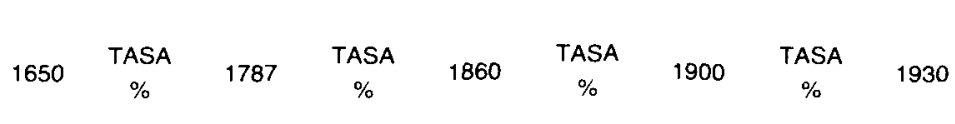

$\begin{array}{lrrrrrrrrr}\text { ESPAÑA } & 6.900 .000 & 0,30 & 10.409 .879 & 0,56 & 15.655 .467 & 0,43 & 18.607 .674 & 0,79 & 23.563 .867 \\ \text { GALICIA } & 820.000 & 0,36 & 1.345 .803 & 0,40 & 1.799 .224 & 0,24 & 1.980 .515 & 0,39 & 2.230 .281 \\ \text { CANARIAS } & 40.000 & 1.05 & 169.285 & 0.46 & 237.036 & 1,04 & 358.564 & 1,46 & 555.128\end{array}$

Si del marco extenso de la larga duración nos trasladamos a la observación más detallada de los incrementos intercensales del periodo 1857-1930 y ampliando la observación al cuadro provincial gallego, nos sería difícil igualmente apreciar cualquier tipo de correspondencia mecánica entre crecimiento demográfico e intensidad migratoria (Pontevedra y La Coruña emiten una emigración relativa muy superior a las de Orense y Lugo), ni tampoco lo contrario.

\begin{tabular}{ccccccccc}
\hline $\begin{array}{c}\text { TASA } \\
\%\end{array}$ & $1858-77$ & $1878-87$ & $1888-97$ & $\begin{array}{c}1898- \\
1900\end{array}$ & $1901-10$ & $1911-20$ & $1921-30$ & $\begin{array}{c}1858- \\
1930\end{array}$ \\
\hline ESPAÑA & 0,36 & 0,54 & 0,31 & 0,89 & 0,69 & 0,67 & 1,01 & 0,58 \\
CORUÑ A ... & 0,40 & 0,29 & 0,28 & 1,15 & 0,34 & 0,46 & 0,80 & 0,46 \\
LUGO $\ldots . .$. & Negativo & 0,51 & 0,60 & 0,45 & 0,30 & Negativo & Negat. & 0,14 \\
ORENSE ... & 0,30 & 0,41 & Negativo & 0,11 & 0,17 & 0,02 & 0,32 & 0,19 \\
PONTEV. ... & 0,15 & Negativo & 0,09 & 0,92 & 0,80 & 0,74 & 0,63 & 0,39 \\
GALICIA & 0,16 & 0,25 & 0,24 & 0,67 & 0,41 & 0,29 & 0,48 & 0,31 \\
CANARIAS & 0,92 & 0,37 & 1,38 & 2,34 & 2,16 & 0,30 & 1,94 & 1,20 \\
\hline
\end{tabular}

En el caso canario, la intensificación o aminoración del éxodo parece influir sobre el crecimiento demográfico interior; y así el período de 185877 , caracterizado como de "relativa detención del flujo emigratorio",

emigración, una estructura en la historia de las Canarias, Las Palmas 1981, p. 15. Las otras cifras para 1650 son estimaciones propias. 
muestra un crecimiento superior al periodo 1878-87 en que la emigración se reactiva por problemas económicos coyunturales ${ }^{12}$. Pero en el caso gallego es curioso observar que, si esto mismo parece cumplirse por ejemplo en el período de considerable emigración 1858-77, no se acusa del mismo modo en la provincia de La Coruña y en la de Pontevedra; o que sólo la provincia de Lugo detiene su crecimiento en la época de intensa emigración de la década de 1920. Puede observarse también que el período 1888-97, en el que las provincias interiores de Galicia se incorporan a la emigración masiva, corresponde en Orense a un periodo de crecimiento negativo (explicado en buena medida por la plaga de la filoxera), pero en Lugo coincide con el decenio de mayor crecimiento de la población. En resumen, todo esto nos indica que la emigración puede ser o no una resultante inmediata del crecimiento demográfico, o bien de otras causas; y que la puesta en funcionamiento del mecanismo regulador migratorio no depende exclusivamente del crecimiento demográfico mismo, sino también de las circunstancias en que dicho crecimiento se origina, entre ellas las densidades de partida, el régimen demográfico mismo, y por supuesto, el sistema económico que sirve de contexto. Nos indica también que la detención del crecimiento demográfico puede sobrevenir o no como consecuencia de la emigración; ya que el régimen demográfico puede absorber sus efectos en muy desigual medida, como se aprecia en los desiguales crecimientos de Galicia y Canarias, con niveles de emigración relativa que pudiéramos considerar provisionalmente como equiparables. Sin olvidar que los efectos de la emigración pueden o no verse compensados por una inmigración de signo contrario (dependiendo esto también de la atracción económica de las respectivas áreas), como puede observarse durante el período 1888-1900 para las principales provincias españolas que envían emigrantes a América, sin perjuicio de lo cual unas presentan saldo migratorio negativo y otras positivo.

${ }^{12}$ Un minucioso examen de esta problemática, en MaClas Hernandez, A.: “Un siglo de emigración canaria, 1830-1930". En SANCHEZ AlBORNOZ, N., op. cit., pp. 166-202. 


\begin{tabular}{|c|c|}
\hline BARCELONA $\ldots \ldots \ldots$ & 94 por 10.000 \\
\hline CADIZ & 16 por 10.000 \\
\hline CANARIAS $\ldots \ldots \ldots \ldots$ & 56 por 10.000 \\
\hline SANTANDER $\ldots \ldots \ldots$ & 5 por 10.000 \\
\hline VIZCAYA & 111 por 10.000 \\
\hline CORUÑA & 29 por 10.000 \\
\hline LUGO .......... & 13 por 10.000 \\
\hline ORENSE & 30 por 10.000 \\
\hline PONTEVEDRA $\ldots$ & 36 por 10.000 \\
\hline VIEDO & 18 por 10.000 \\
\hline
\end{tabular}

Lo que sí puede decirse es que en el caso gallego la emigración a América funcionó como válvula reguladora para frenar parcialmente su crecimiento demográfico, en función de un saldo migratorio permanentemente negativo. Incluso en el período 1888-1900, en el que hay que tener en cuenta los retornos producidos por la guerra de Cuba y pérdida de las colonias, el saldo migratorio es netamente negativo para todas las provincias gallegas. La diferencia con respecto a Canarias no se debe sólo a la inexistencia de cualquier corriente de inmigración hacia Galicia, sino también a las diferencias del régimen demográfico y de su potencialidad intrínseca para generar excedentes vegetativos ${ }^{13}$ :

${ }^{13}$ Las dos tablas referidas a las provincias gallegas y otras tienen por fuente la Estadistica de la Emigración e Inmigración de España en el quinquenio de 1896-1900. Madrid 1903. 


\begin{tabular}{|c|c|c|c|}
\hline PERIODO 1888-1900 & $\begin{array}{c}\text { NATALIDAD } \\
\%\end{array}$ & $\begin{array}{c}\text { MORTALIDAD } \\
\%\end{array}$ & $\begin{array}{c}\text { SALDO } \\
\text { VEGETATIVO } \\
\%\end{array}$ \\
\hline$\ldots \ldots \ldots \ldots \ldots \ldots$ & 36 & 31 & 5 \\
\hline$\ldots \ldots \ldots \ldots \ldots$ & 33 & 25 & 8 \\
\hline LUGo $\ldots . . . . . . . . . . . . . . . .$. & 34 & 27 & 7 \\
\hline ORENSE $\ldots \ldots \ldots \ldots \ldots \ldots \ldots \ldots$ & 31 & 28 & 3 \\
\hline PONTEVEDRA $\ldots \ldots \ldots \ldots \ldots \ldots$ & 30 & 23 & 7 \\
\hline GALICIA & 32 & 26 & 6 \\
\hline CANARIAS & 34 & 23 & 11 \\
\hline
\end{tabular}

A diferencia de Canarias, cuyo crecimiento vegetativo duplica a la media española, el crecimiento vegetativo de Galicia no permite explicar el fenómeno diferencial de su emigración respecto al conjunto español, pues es similar a éste. Queda claro por tanto que la emigración en Galicia no se justifica ni en razón del crecimiento real de su población ni de su crecimiento vegetativo. La presión demográfica vendría aqui determinada por una superpoblación relativa, que debe razonarse como una densidad de población excesiva para sus recursos; conclusión que no hace sino trasladar más atrás en el tiempo la responsabilidad del crecimiento demográfico, ya que las altas densidades de la población gallega en los siglos XVIII-XIX son el resultado de los fuertes crecimientos experimentados en el siglo XVI y principalmente en el $\mathrm{XVII}^{14}$.

Pero, al igual que ocurre con el crecimiento real y natural de la población, tampoco la densidad de población aisladamente considerada es un valor que por si mismo baste para razonar el fenómeno migratorio. La tabla que recoge las densidades de población y los indices migratorios

14 En 1591 las densidades de Galicia y Castilla son de 22 y 18 habitantes por kilómetro cuadrado respectivamente; y a finales del XVII de 34 y 15 respectivamente. Partimos para 1591 de la cifra convencional dada por Ruiz ALMANSA; y para 1700 de nuestras propias estimaciones sobre la población de Galicia y de Castilla. 
(emigración relativa por cada 10.000 habitantes) de las 20 provincias españolas con mayor emigración relativa a América, demuestra que ambos valores guardan poca o ninguna correlación entre si ${ }^{15}$.

NÚMERO DE EMIGRANTES DEL QUINQUENIO

\begin{tabular}{|c|c|c|c|c|c|}
\hline PERIOODO 1891-95 & CONTINENTE & COLONIAS & AMÉRICA & $\begin{array}{c}\text { ANUAL } \times \\
10.000 \mathrm{~h} .\end{array}$ & DENSIDAD \\
\hline CANARIAS $\ldots \ldots \ldots \ldots \ldots$ & 2.701 & 17.808 & 20.509 & 140 & 40 \\
\hline PONTEVEDRA $\ldots \ldots \ldots \ldots$ & 19.949 & 7.093 & 27.042 & 122 & 102 \\
\hline CORUÑA $\ldots \ldots \ldots \ldots \ldots$ & 9.139 & 24.760 & 33.899 & 110 & 77 \\
\hline ORENSE $\ldots \ldots \ldots \ldots \ldots$ & 9.110 & 6.431 & 15.541 & 77 & 58 \\
\hline OVIEDO $\ldots \ldots \ldots \ldots \ldots \ldots$ & 3.673 & 18.367 & 22.040 & 74 & 54 \\
\hline SANTANDER $\ldots \ldots \ldots \ldots$ & 1.876 & 4.387 & 6.263 & 51 & 46 \\
\hline LUGO $\ldots \ldots \ldots \ldots \ldots \ldots$ & 1.397 & 8.766 & 10.163 & 47 & 44 \\
\hline BARCELONA $\ldots \ldots \ldots \ldots$ & 5.176 & 15.019 & 20.735 & 46 & 117 \\
\hline CÁDIZ . & 1.560 & 7.759 & 9.319 & 43 & 58 \\
\hline MÁlAGA $\ldots \ldots \ldots \ldots \ldots \ldots$ & 7.349 & 146 & 7.495 & 29 & 71 \\
\hline LEON $\ldots \ldots \ldots \ldots \ldots \ldots$ & 1.756 & 2.357 & 4.113 & 21 & 27 \\
\hline VIZCAYA $\ldots \ldots \ldots \ldots \ldots$ & 792 & 1.661 & 2.453 & 21 & 106 \\
\hline VALLADOLID $\ldots \ldots \ldots \ldots$ & 177 & 2.453 & 2.630 & 19 & 32 \\
\hline BALEARES $\ldots \ldots \ldots \ldots \ldots$ & 458 & 2.103 & 2.561 & 16 & 62 \\
\hline GRANADA $\ldots \ldots \ldots \ldots \ldots$ & 3.941 & 77 & 4.018 & 16 & 39 \\
\hline GERONA $\ldots \ldots \ldots \ldots \ldots$ & 473 & 1.851 & 2.324 & 15 & 74 \\
\hline ZARAGOZA $\ldots \ldots \ldots \ldots$ & 656 & 2.481 & 3.137 & 15 & 24 \\
\hline TARRAGONA $\ldots . . .$. & 576 & 1.333 & 1.909 & 11 & 55 \\
\hline GUIPÚZCOA $\ldots$. & 404 & 465 & 869 & 10 & 91 \\
\hline MADRID $\ldots \ldots \ldots \ldots \ldots$ & 1.173 & 2.012 & 3.185 & 10 & 85 \\
\hline ESPAÑA $\ldots \ldots \ldots \ldots \ldots$ & 79.418 & 131.919 & 211.337 & 24 & 35 \\
\hline GALICIA . & $\begin{array}{l}39.595 \\
(50 \%)\end{array}$ & $\begin{array}{l}47.050 \\
(35 \%)\end{array}$ & $\begin{array}{l}86.645 \\
(41 \%)\end{array}$ & 91 & 64 \\
\hline
\end{tabular}

Se comprueba que la intensidad de la emigración no es mera función matemática de la densidad de población ni nada parecido a eso. Lugo

15 FUENTE: Estadistica de la Emigración e Inmigración de España en el quinquenio 1891-1895. Madrid 1898. 
coincide con Santander en densidad de población y también en intensidad migratoria; y lo mismo ocurre entre Orense y Oviedo. Pero Lugo tiene la misma densidad que las Canarias, y éstas triplican su intensidad migratoria. A su vez, Lugo coincide en intensidad migratoria con Barcelona, pero ésta la triplica en densidad. Orense se equipara en densidad a Baleares y a Tarragona, pero las quintuplica o sextuplica en intensidad migratoria. Coruña se iguala en densidad con Málaga y con Gerona, pero las cuadruplica o sextuplica por la intensidad de su emigración; tiene una densidad inferior a Guipúzcoa, y sin embargo su proporción de emigrantes es diez veces más alta. Pontevedra se equipara en densidad a Barcelona y a Vizcaya, pero su intensidad de emigración es tres veces superior a la primera y seis veces superior a la segunda.

Aquí es en donde entra en juego el sistema económico y social. Dentro de un contexto social y económico uniforme la densidad de población (que en tal caso viene a ser una variable dependiente de los determinantes geográficos) sí parece correlacionarse con el nivel de la emigración: así la intensidad migratoria de las cuatro provincias gallegas sigue un orden riguroso de gradación que se corresponde con el de sus respectivas densidades de población (Pontevedra, Coruña, Orense y Lugo) ${ }^{16}$. Lo mismo parece encontrarse en el interior de los conjuntos constituidos por las provincias catalanas y por las vascas. Con densidades de población muy próximas a las de Galicia, en Cataluña y en el Pais Vasco hallamos tasas de emigración relativa mucho más bajas, aun tratándose de pueblos con una larga tradición emigrante a América, que en Cataluña se remonta al siglo xVIII, y en el caso de los vascos a los albores mismos del Descubrimiento. Lo que en esta diferencia se aprecia es el contraste entre la economía enteramente primaria de Galicia, generadora de emigración, y las industrializaciones catalana y vasca, capaces de moderar su emigración, e incluso de provocar saldos migratorios positivos, como más atrás se ha visto para todo el periodo 1888-1900 ${ }^{17}$. Una mirada a lo que ocurre en los paises europeos que

${ }_{16}$ Aunque con cierta desproporción en los casos de Coruña y Orense, que deben explicarse por razones coyunturales. Esto es claro en el caso de la provincia de Orense, afectada en este periodo por la crisis de la filoxera. En el caso coruñés habría que pensar tal vez en motivos de crisis industrial, como la que sufren los astilleros ferrolanos en este momento. Vid. Palomares ibánez, J. M y Fernández Casanova, M. C.: la Comisión de Reformas Sociales y la Cuestión Social en Ferrol (1184-1903). Univ. de Santiago, 1984, 202 páginas.

17 Esto hace más patente el desfase de algunos organismos públicos españoles que, todavia a finales del sigio xIX, se oponen a la emigración con una política populacionista de tipo mercantilista tradicional, y que pretenden corregir sus causas mediante soluciones ana- 
emigran a América en el siglo xIX puede dejar aún más claro: véase tabla de drenaje migratorio para el año $1988^{18}$.

\begin{tabular}{|c|c|c|c|c|c|}
\hline AÑo 1898 & $\begin{array}{l}\text { DENSIDAD } \\
\text { POBLACION }\end{array}$ & $\begin{array}{l}\text { INCREMENTO } \\
\text { NATURAL }\end{array}$ & $\begin{array}{l}\text { EMIGRACION } \\
(\%)\end{array}$ & $\begin{array}{l}\text { CRECI- } \\
\text { MIENTO } \\
\text { REAL }\end{array}$ & $\begin{array}{c}\text { DRENAJE } \\
\text { MIGRA- } \\
\text { TORIO } \\
(\%)\end{array}$ \\
\hline IRLANDA $\ldots$. & 54 & 5,07 & 7,56 & $-2,49$ & 149,11 \\
\hline ITALIA $\ldots \ldots \ldots \ldots \ldots \ldots$ & 110 & 10,70 & 4,40 & 6,30 & 41,12 \\
\hline ESPAÑA $\ldots \ldots \ldots \ldots \ldots$ & 36 & 5,14 & 1,82 & 3,32 & 35,40 \\
\hline ESCOCIA $\ldots \ldots \ldots \ldots \ldots$ & 54 & 12,35 & 3,66 & 8,69 & 29,63 \\
\hline INGLATERRA-GALES $\ldots \ldots$ & 207 & 11,82 & 2,88 & 8,94 & 24,36 \\
\hline FRANCIA $\ldots \ldots \ldots \ldots \ldots$ & 71 & 0,89 & 0,15 & 0,74 & 16,85 \\
\hline NORUEGA $\ldots \ldots \ldots \ldots \ldots$ & 7 & 15,01 & 2,27 & 12,74 & 15,12 \\
\hline AUSTRIA $\ldots \ldots \ldots \ldots \ldots$ & 84 & 11,28 & 1,33 & 9,95 & 11,79 \\
\hline HUNGRIA $\ldots \ldots \ldots \ldots \ldots$ & 58 & 9,68 & 1,02 & 8,66 & 10,53 \\
\hline DINAMARCA $\ldots \ldots \ldots \ldots$ & 59 & 14,89 & 0,99 & 12,90 & 6,64 \\
\hline SUIZA $\ldots \ldots \ldots \ldots \ldots \ldots$ & 75 & 10,54 & 0,54 & 10,00 & 5,12 \\
\hline ALEMANIA $\ldots \ldots \ldots \ldots$ & 100 & 15,58 & 0,40 & 15,18 & 2,56 \\
\hline BELLICA $\ldots \ldots \ldots \ldots \ldots$ & 226 & 11,08 & 0,14 & 10,94 & 1,26 \\
\hline paÍsES BAJOS $\ldots \ldots \ldots \ldots$ & 152 & 14,88 & 0,16 & 14,72 & 1,07 \\
\hline GALICIA $\ldots \ldots \ldots \ldots \ldots$ & 64 & 6,14 & 2,90 & 3,24 & 47,23 \\
\hline
\end{tabular}

Una vez más se comprueba que ni la densidad de población, ni el crecimiento natural o real de la población, aisladamente considerados, guardan ningún tipo de correlación fija con la intensidad de emigración (expresada como emigración relativa por mil habitantes). Irlanda e Italia ofrecen la más alta emigración relativa, pero la una con una densidad de tipo medio y la otra con una densidad muy elevada; mientras por el extremo contrario Holanda y Bélgica presentan la mínima emigración relativa con densidades

crónicas de crecimiento agrario extensivo de tipo primario. Reflejando la mentalidad oficial del momento, los autores de la Estadistica de la Emigración e Inmigración de España en el quinquenio 1896-1900 (p. XXI) proponen absorver la "avalancha de reimpatriados" que retornan de Cuba, Puerto Rico y Filipinas mediante soluciones tales como "roturar las grandes masas de tierra inculta" o "colonizar los extensos eriales desiertos".

${ }^{18}$ Elaboración a partir de datos publicados en la Estadistica citada en la nota anterior. En la columna de "drenaje migratorio" consignamos el porcentaje de la emigración sobre el saldo vegetativo de cada pais. 
de población muy superiores a las de Italia. Alemania y Noruega se sitúan a la cabeza de los países europeos por su incremento natural y real, pero sus emigraciones relativas son muy diferentes. La única correlación más o menos clara puede percibirse entre las dos últimas columnas de la tabla: en este caso una correlación negativa entre crecimiento real y proporción de drenaje migratorio (llamando asi al porcentaje del crecimiento vegetativo que cada país expulsa a la emigración). Vale decir que los países que crecen más rápidamente en población son también generalmente los que retienen dentro de si la práctica totalidad de su crecimiento vegetativo, por ser económicamente capaces de asumirlo. $Y$ aquí tocamos la clave del asunto. La lista de países de la tabla anterior, ordenados de mayor a menor proporción de drenaje migratorio, viene a dar algo muy próximo a una relación de países ordenados de menor a mayor grado de desarrollo económico. Los países desindustrializados (España, Italia y el caso límite de Irlanda ofrecen crecimientos de población muy bajos y se sitúan en los primeros lugares de la tabla por su proporción de drenaje migratorio, que en el caso límite de Irlanda llega a superar a su incremento natural. Por el otro extremo, los países de industrialización reciente y crecimiento económico muy acelerado (Dinamarca, Suiza, Alemania, Bélgica y Holanda) soportan los más rápidos crecimientos demográficos mientras sus proporciones de drenaje migratorio son irrelevantes. En posiciones intermedias entre ambos extremos está un grupo heterogéneo de países en situaciones diversas, 0 bien con una incipiente modernización económica (Noruega, Austria, Hungria), o bien con una industrialización muy temprana y que a finales del XIX están agotando las posibilidades de asimilar su rápido crecimiento natural (Inglaterra, Gales, Escocia); en el grupo entra también el caso particular y malthusiano de la Francia del crecimiento cero, fenómeno peculiar bien conocido para los historiadores de la población. La conclusión parece clara: cuanto más retrasado en su evolución económica es un pais, mayor es el porcentaje de su saldo vegetativo que envía a la emigración. Esto es lo único que puede afirmarse; ya que el volumen absoluto de la emigración, e incluso la misma migración relativa, en un país subdesarrollado pueden quedar aminorados por una débil capacidad de excedentes de su régimen demográfico, como revela el caso de España al compararla con Italia. Volviendo ya a Galicia, por su proporción de drenaje migratorio se situaría en la cabeza de la tabla, por encima de España y de Italia y sólo detrás de Irlanda. Esto coloca a Galicia a la cabeza de los países desindustrializados y de estructura económica arcaica ${ }^{19}$. Sólo Irlanda

\footnotetext{
${ }^{19}$ Con un saldo vegetativo $(6,14$ por 1.000$)$ similar al de Irlanda o de España; una tasa
} 
entre los países europeos conocidos estadísticamente muestra un resultado más desfavorable y una más baja eficiencia de su sistema económico; aunque no puede dudarse de que existan otras regiones europeas cuyos datos estadísticos no nos son conocidos y que pudieran relevar a Galicia del dudoso honor de encabezar la tabla de sistemas ineficientes.

Las comparaciones realizadas hasta aquí parecen demostrar que en Galicia no se da un grado de presión demográfica que por sí misma justifique la intensidad de su emigración, ni al cotejarla con el conjunto de las regiones españolas, ni menos aún con el resto de los países europeos. En la época considerada Galicia tiene la misma densidad que Escocia o Dinamarca, pero expulsa a la emigración una proporción de dos a siete veces superior de su saldo biológico natural. Galicia sostiene un crecimiento vegetativo inferior al de Suiza o al de Noruega, y sin embargo debe segregar una parte de él de tres a diez veces superior. Es verdad que Galicia presenta una tasa neta de emigración similar a la de Inglaterra; pero este país triplica a Galicia en densidad de población y la duplica en cuanto a su dinamismo demográfico, que en Galicia es muy débil y no autoriza a hablar de una especial "fecundidad de la raza" ${ }^{20}$. Los moderados parámetros de la demografía gallega en el período 18881900 pueden generalizarse a toda la etapa estadística que precede a la Gran Guerra (1857-1914), en la que los excedentes naturales de Galicia difieren muy poco de los del conjunto español ${ }^{21}$.

Por estos vericuetos, el análisis realizado a partir de las estadísticas demográficas nos parece que traslada la causa real de la emigración de un sistema arcaico de economía primaria de pequeños agricultores de subsistencia, que perdura en Galicia como una reliquia del sistema económico preindustrial. Esta constatación nos parece de un cierto interés, porque nos exigirá en adelante considerar la emigración gallega no sólo en su aspecto demográfico, sino también en su aspecto económico; toda vez que, según las conclusiones a que acabamos de llegar, sus motiva-

de emigración neta (2,90 por 1.000) intermedia entre las de España e Italia; y una proporción de drenaje migratorio $(47,23$ por 100$)$ de tipo italiano más que español.

${ }_{20}$ Recordemos que para el período $1888-1900$ el saldo vegetativo de Galicia es igual al medio español y sólo la mitad que el de Canarias. La tasa de natalidad es sólo de 32 por 1.000 , frente a 34 por 1.000 en Canarias y 36 por 1.000 en el conjunto español.

${ }_{21}$ Resultados del periodo 1857-1914: en tantos por mil. Natalidad: España 34,85; Galicia 31,11. Mortalidad: España 28,72; Galicia 23,32. Saldo vegetativo: España 6,13; Galicia 7,79. Datos tomados de LOPEZ TABOADA, X. A.: Economía e población en Galicia, 1979, p. 132. 
ciones son en parte demográficas, pero lo son aún más económicas. Desde su componente demográfico, la función de la emigración gallega queda bastante clara: moderar el crecimiento de la población evitando que se dispare y manteniéndolo en niveles más bajos que los del conjunto español ${ }^{22}$. Grosso modo, la función económica de la emigración debió ser la de producir una parte de los recursos no generados por el sistema productivo para compensar las precariedades de una economía deficitaria y sometida a un proceso de permanente descapitalización por la doble vía del impuesto y la renta. También aquí hallamos otro punto de analogía ya anticipado por la historiografía canaria ${ }^{23}$. Aunque todavía insuficientemente estudiado, el tema de la repatriación de capitales indianos y, principalmente, de la llegada y orientación de las remesas de emigrantes, comienza a ser conocido gracias a algún estudio reciente ${ }^{24}$. Las primeras noticias hasta ahora reunidas sobre remesas de emigrantes gallegos datan sólo de $1861^{25}$. Pero es seguro que desde mucho tiempo antes los emigrantes gallegos enviaban sus ahorros a la tierra natal, como ocasionalmente nos informan las escrituras notariales. En una primera fase, y antes de la creación de los primeros circuitos bancarios para el giro de remesas (Banco español del Río de la Plata, Banco de Comercio Hispano-Argentino, Banco Hispano-Americano, etc.) el envío de ahorros era canalizado a través de los cónsules, o de los agentes oficiales de inmigración establecidos - desde 1870, al menos en el caso de la República Argentina- en Galicia; y muy probablemente también a través de los mismos armadores gallegos, que hasta la década de 1870 realizaban el transporte de los emigrantes ${ }^{26}$. Desde finales del XIX y principios del $x x$, coincidiendo con un casi seguro cambio cualitativo en la duración

${ }^{22}$ Así en 1888-1900 el crecimiento demográfico gallego fue de 0,34 por 100 acumulativo anual frente al 0,45 por 100 en el conjunto español. Sin la emigración el español habría sido del 0,52 por 100 anual, y el gallego habria sufrido hasta el 0,60 por 100 anual, colocándose por encima.

${ }^{23}$ Las islas Canarias padecen históricamente una "permanente descapitalización" efecto de una balanza comercial estructuralmente deficitaria. Una función de la emigración canaria fue la de cubrir este déficit a través de las "remesas invisibles" de los emigrantes a sus familias y de los capitales repatriados por los indianos. Cf. BETHENCOURT, A., op. cit. p. 20.

${ }^{24}$ VAZZuez Gonzalez, Alejandro: "La emigración gallega. Migrantes, transportes y remesas", en SÁnchez Albornoz, Nicolás, op. cit. nota 1, pp. 80-104. Las remesas de emigrantes recibidas en la comarca de Chantada durante el primer tercio del siglo xx han sido estudiadas por VilLARES PAZ, Ramón: La propiedad de la tierra en Galicia (1500-1936), Madrid 1982, pp. 396-401.

${ }_{25}$ Hasta los primeros años de nuestro siglo las pocas cifras disponibles nos dan la impresión de ser muy incompletas. vid. VAZqueZ GonZÁLEZ, Alejandro, op. cit. p. 96.

${ }^{26}$ La transformación de la casa armadora de José Pastor, de La Coruña, en la Casa de Banca Sobrinos de José Pastor induce a suponerlo así. 
de la emigración, el establecimiento de redes bancarias estables facilitó no sólo la seguridad de los envíos, sino también el conocimiento de su volumen ${ }^{27}$.

Son variados los cauces a través de los cuales el dinero ganado en la emigración pudo penetrar en la economía gallega y contribuir a liberar su décifit estructural. En principio el dinero de los emigrantes viene dirigido a la mera resolución de obligaciones pendientes (liquidación del pasaje a crédito, liberación de deudas usurarias) o bien al sostenimiento de la pequeña explotación agraria (aumento del ganado, adquisición plena del ganado en aparcería, adquisición de parcelas, reparación de instalaciones domésticas $o$, en fin, rendención de rentas forales). Sobre este último punto, puede que convenga precisar en el tiempo la idea de que la redención de rentas forales fue el destino prioritario de los ahorros de los emigrantes. En el primer tercio de nuestro siglo no puede dudarse de que así haya sido, principalmente en la coyuntura histórica de la redención legal del foro ${ }^{28}$. Antes del siglo $\mathrm{xx}$, sin embargo, sobre no estar creadas las condiciones legales para la redención foral -fuera del fugaz paréntesis de 1873, que por su brevedad apenas permitió obtener algún resultado práctico en esta materia-, cabe poner en duda que este objetivo pudiera entrar en las prioridades del campesino emigrante. En nuestra opinión, debió ser prioritario para él liberar primeramente el ganado en aparceria, por el que venía pagando a las burguesias propietarias — rural y urbana - cánones de renta próximos al 50 por 100 del producto neto ganadero, mientras que los cánones de la renta foral eran generalmente mucho más bajos. Un estudio sobre la desaparición o no de la vieja y universal fórmula de la aparcería de ganado en Galicia ("ganado a la ganancia») a la altura del 1900 debería confirmar esta hipótesis. En todo caso, si bien el destino próximo del dinero de los emigrantes eran las propias necesidades inmediatas de la propia explotación agraria del minifundio - añadiendo tal vez a la lista anterior todavía el pago de las contribuciones en muchos casos-, este dinero ingresaba en el circuito de la economía general, a través del armador o de la casa consignataria a la que se pagaban los

${ }^{27}$ Para los primeros años del siglo $x x$ se aprecia ya concordancia en las cifras sobre remesas totales recibidas en Galicia desde la Argentina, a partir de datos del Banco Español del Río de la Plata; así entre los 40 a 50 millones de pesetas que menciona GonzALEz BESADA, Augusto, para 1904 y los 48 millones de pesetas que recoge VICENTI, Eduardo, para 1906. Cit. por Alejandro VAZOUEZ GonzÁlez, op. cit. p. 96.

${ }^{28}$ VILLARES, R., op. cit., pp. 401-405, se plantea la cuestión de la dedicación de remesas de América a la redención de rentas forales en el primer tercio del siglo. Así parece ser el caso en la comarca de Chantada en torno a la década de 1920. 
pasajes y los intereses del crédito de los mismos; del burgués al que se liquidaba la propiedad del ganado o el pago de la deuda; y finalmente, de los propietarios a los que se les redimieron los foros. Por otra parte, se ha señalado la aparición de algunos núcleos capitalistas (Banco Pastor, Banco Olimpio Pérez, etc.), principalmente en los grandes puertos de embarque de las cohortes de emigrantes, cuyo origen se encuentra en las mismas casas armadoras o consignatarias de la emigración, que más tarde diversifican sus actividades y reinvierten sus beneficios en empresas de variada indole ${ }^{29}$.

Parece llamada a afianzarse la idea de que la emigración gallega experimentó un cambio en su orientación, pasando a realizar una función más bien de regulación demográfica, la de contribuir a aligerar la presión de la población sobre los recursos, a una función más económica, la de producir pequeños capitales para las inversiones ya expresadas ${ }^{30}$. La fase de tránsito estaría situada en la última década del XIX y primera del $x \mathrm{X}$, en relación con la reducción de la duración de los viajes y el abaratamiento real de los pasajes, que empieza a percibirse entonces después de las dos décadas de serio enrarecimiento de los transportes que acompaña a la llegada de la navegación a vapor ${ }^{31}$. Este doble cambio favoreció un retorno más fluido de los emigrantes y estimuló una nueva emigración por períodos más cortos. Los porcentajes de retorno de emigrantes a puertos gallegos acusan este cambio, pasando de sólo un 25 por 100 de retornos hasta finales de la década de 1880 a un 45 por 100 de retornos en el quinquenio 1890-95, y a más del 50 por 100 de retornos en el período $1900-1913^{32}$. En principio, estos datos permiten generalizar también el caso gallego la tesis de un incremento de la emigración "golondrina" desde los años finales del siglo $\mathrm{XIX}^{33}$. Esta figura se encuentra bien documentada a partir de las mismas fechas en el caso de la emigración canaria, relacionándola con la dedica-

29 VÁzouez Gonzalez, Alejandro, op. cit., p. 103.

${ }^{30}$ LÓPEZ TABOADA, X. A., op. cit., pp. 67-68.

${ }^{31}$ Se ha comprobado un abaratamiento del precio de los pasajes de las Canarias a América desde la década de 1890 hasta el comienzo de la Guerra Europea. Cf. HernANDEZ GARCiA, J., pp. 305-308. Este efecto de la competencia y del progreso técnico no pudo dejar de tener su equivalente en la navegación a partir de Galicia, ya que las navieras internacionales eran las mismas, que pasando por Galicia hacian escalas en las islas Canarias.

${ }_{32}$ LOPEZ TABOADA, X. A., op. cit., pp. 153-154. Los retornos se acercan al 100 por 100 en los años 1897-99; pero este hecho bien conocido y generalizado es mera consecuencia de la guerra de Cuba y de la pérdida de las colonias tras el desastre del 98.

33 SÁNCHEZ ALONSO, Blanca: “La emigración española a la Argentina, 1880-1930", en SánChez Albornoz, N., op. cit., nota 1, pp. 205-235. Díaz Melián, Mafalda V.: La inmigración española en la década del ochenta, Buenos Aires, 1980, p. 127, sitúa más precisamente entre 1892 y 1904 el comienzo de la emigración "golondrina" a la Argentina. 
ción agrícola de los emigrantes temporeros, y más concretamente con las labores de la zafra ${ }^{34}$. A partir de la lógica de las facilidades de las comunicaciones y el beneficio diferencial de los salarios, se ha pensado que el incremento de los porcentajes de retorno que registra simultáneamente la emigración gallega puede corresponder igualmente a un incremento de la emigración "golondrina". Tal vez el caso gallego exija un estudio más profundizado sobre este punto, ya que se parte aquí de algunas diferencias importantes con el caso canario, como son el alto porcentaje de emigración gallega a la Argentina, la mayor distancia de los trayectos, principalmente al Río de la Plata, y el hecho de que la emigración "golondrina" en la Argentina tuvo siempre una importancia mucho menor que en Cuba. Pero la idea fundamental de un cambio cualitativo de la emigración gallega desde fines del XIX, ya se trate de auténtica emigración "golondrina", o de una nueva emigración de ciclo corto (por dos o más años), parece acreditado por las simples cifras de los porcentajes de retorno, al igual que su consecuencia de mantener desde entonces al emigrante más ligado a la tierra de procedencia.

${ }^{34}$ HeRnández García, J., op. cit., pp. 513-514. Un estudio más pormenorizado de este tema, en A. Macías HeRnández, op. cit., 186-198. 